\title{
RF CMOS Low Noise Amplifier Design-A Case Study R.K.Lamba ${ }^{a^{*}}$, C.H.Vithalani ${ }^{\mathrm{b}}$ \\ ${ }^{a}$ Research Student,52-Om Residency,Nana Mava Road, Rajkot, Gujarat 360005, India \\ ${ }^{b}$ Assoc. Prof. \& Head,Dept. of E\&C, Govt. Engg. College, Kankot, Rajkot, Gujarat 360005, India
}

\begin{abstract}
A design methodology of Differential Design of CMOS low noise amplifier (LNA) with source degeneration for Bluetooth frequency is presented. The results show that the proposed topology is effective and can be used to achieve the minimum noise figure at all frequencies of interest. This LNA was realized in $0.18 \mu \mathrm{m}$ CMOS technology using Microwave Office Tool from AWR Inc. The measured noise figure is $2.066 \mathrm{~dB}$ and the gain is $20.29 \mathrm{~dB}$.
\end{abstract}

Index Terms: LNA, Bluetooth, Source Degeneration.

(C) 2014 Published by MECS Publisher. Selection and/or peer review under responsibility of the Research Association of Modern Education and Computer Science

\section{Introduction}

An irreplaceable component of any RF receiver is the front-end low-noise amplifier (LNA). As the first active building block in the receiver front-end, the LNA should provide considerable gain while minimizing the noise introduced to the system. Fig. 1 [6] depicts the simplified structure of an RF receiver front end. As can be seen, the first step of signal amplification is done by the LNA. Therefore, the performance of LNA can greatly affect sensitivity and noise performance [11] of the overall receiver.

\footnotetext{
* Corresponding author. R.K.Lamba Tel.:+91 9825379729

E-mail address: rkls@ rediffmail.com
} 


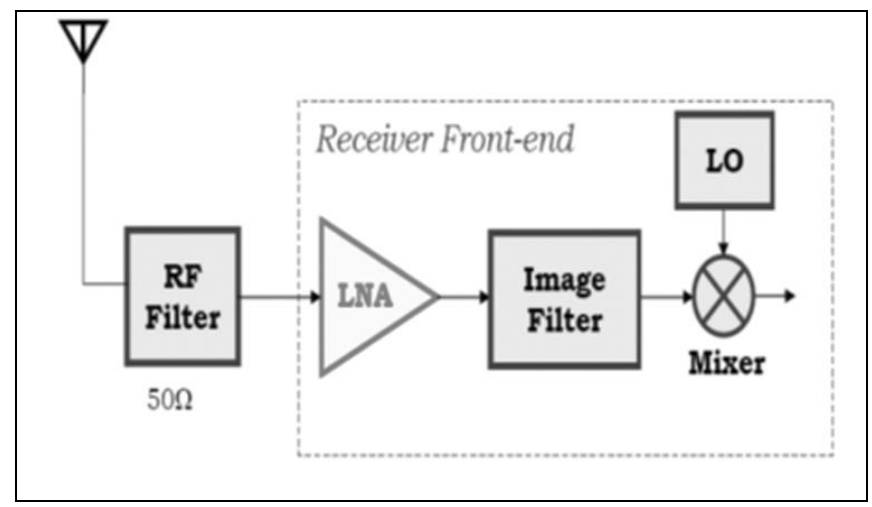

Fig. 1. Receiver Front End

Since the overall noise factor of the receiver front end is dominated by the first stage and can be approximated according to the Frii's formula, [1]

$$
N F_{\text {recfront }}=\left(\frac{1}{G_{\ln a}}\right)\left(N F_{\text {subseuent }}-1\right)+N F_{\ln a}
$$

Where $\mathrm{NF}_{\text {subsequent }}$ is the total input noise factor of the components following the LNA. $\mathrm{G}_{\operatorname{lna}}$ and $\mathrm{NF}_{\operatorname{lna}}$ are the gain and noise factor, of the LNA itself. The noise of all subsequent stages is reduced by the gain of LNA and also the noise of LNA is injected directly into the received signal. Thus LNA needs both, high gain and low noise. [7].

\subsection{Topology}

The general topology of any LNA can be divided into three stages: an input matching network, the amplifier itself and an output matching network. [1].

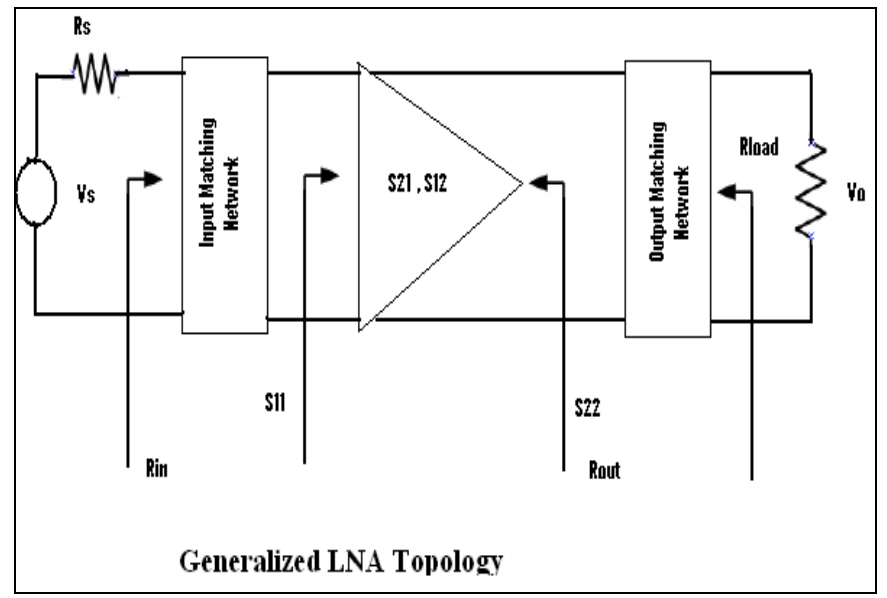

Fig.2. LNA topology 
Depicted in the figure 2 are matching networks and s-parameters, which play a significant role in analyzing the performance on Low Noise Amplifier.

The four $\mathrm{S}$ parameters are -

$\mathrm{S}_{21}=$ Forward gain, Af.

$\mathrm{S}_{12}=$ Reverse transmission (or leakage) factor, Ar. This is usually very small in low frequency, but can become significant at high frequency.

$\mathrm{S}_{11}=$ Input impedance, Rin, $\mathrm{S}_{22}=$ Output impedance, Rout.

The whole design process is carried using the lumped parameters [1], and then the s-parameters are calculated.

To achieve the goal of this paper, out of several topologies, the differential topology [4][6] has been adopted so as to remove the sensitivity to the inductances and to minimize the common mode signal which is described in next section. This section is followed by the calculations of various inductor values and transistor widths for the given gate length and frequency. All these calculations are tabulated in section 4 with a detailed discussion. After this, simulation results have been shown to validate the design. Results for noise figure, gain and sparameters have been included. Dependency of gain and noise figure on inductors has also been shown. At the end, result summary, showing the comparison with references has been included.

\section{Differential LNA}

This section describes the theory and design of a CMOS Differential Low noise amplifier. The Design procedure describes the design of LNA using source degeneration technique to provide a good noise match, improved gain \& reverse isolation. There are several advantages in using a differential design. Firstly, the virtual ground formed at the 'tail' removes the sensitivity to parasitic ground inductances, which makes the real part of the input impedance purely controlled by the source degeneration inductance (Ls).Secondly the differential amplification of the signal ensures attenuation of the common mode signal. The aim of this paper is to design LNA to work over the Bluetooth frequency band. A summary of the required specification for the LNA is given in Table below.

Table 1. Specifications [2]

\begin{tabular}{lll}
\hline Parameter & Specification & Unit \\
\hline Frequency & 2.45 to 2.85 & $\mathrm{GHz}$ \\
& & \\
\hline Noise Figure & $<3.5$ & $\mathrm{~dB}$ \\
\hline Voltage gain & $>20$ & $\mathrm{~dB}$ \\
\hline Power gain & $>10$ & $\mathrm{~dB}$ \\
\hline Source /Load impedance & 50 & $\mathrm{Ohm}$ \\
& & $\mathrm{pF}$ \\
\hline Load Cap. & 0.4 &
\end{tabular}

The design of LNA is carried using Microwave Office from AWR. 


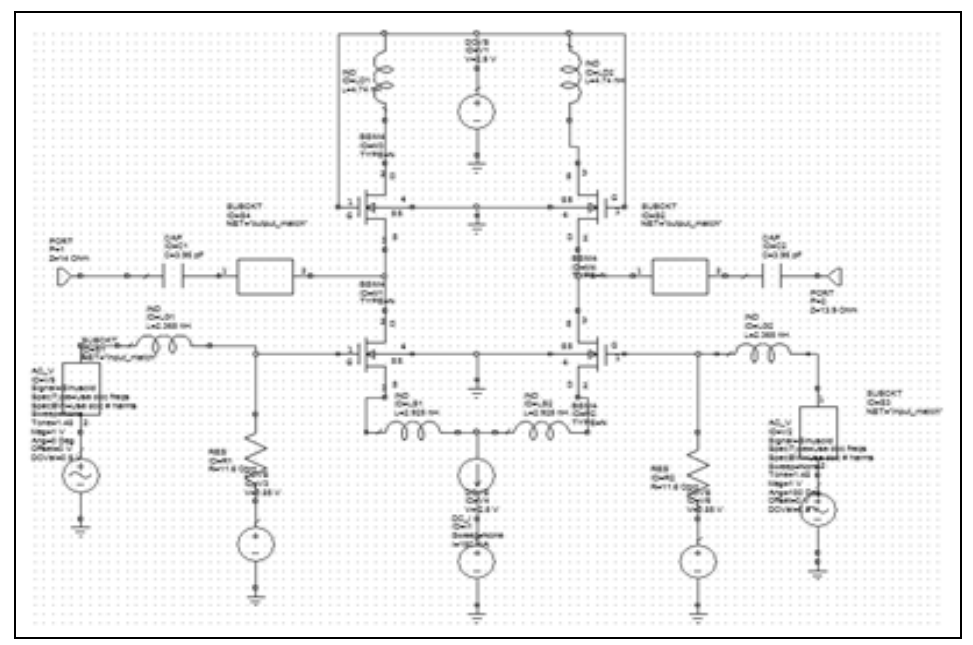

Fig. 3. Differential LNA Design

The inductive degeneration topology is used for impedance matching. [2][5] [15]

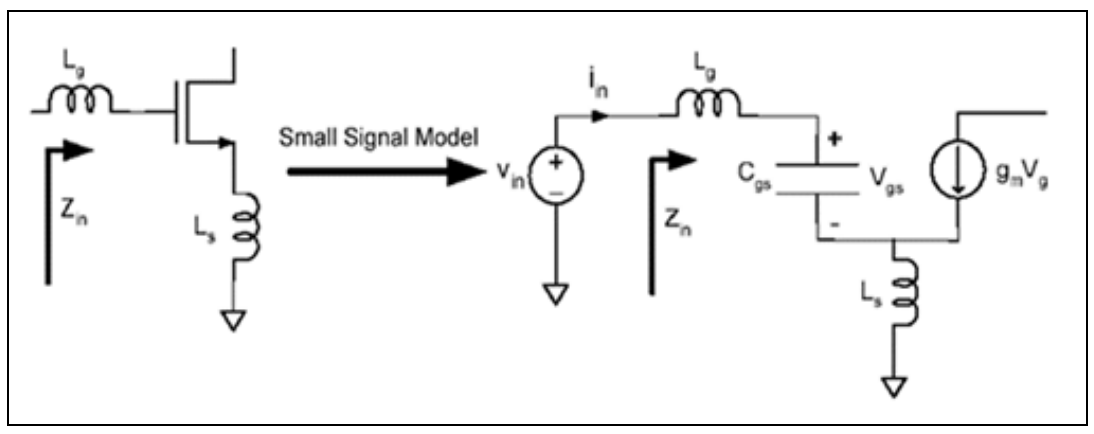

Fig. 4. Small Signal Analysis (Inductive Degeneration)

Referring figure 4 for various parameter calculations[2] the design equations are summarized below:

$$
R_{i n}=R_{g}+\left(\frac{L_{s} g_{m}}{C_{g s}}\right)+j\left(w L s-\frac{1}{w C g s}\right){ }_{\mathrm{Rs}=50 \Omega}
$$

\section{Calculations}

\subsection{Calculation of $L g$ and $L s$}

An another inductor $\mathrm{Lg}$ is added in series with the gate to resonate with the Cgs Capacitor. We have ,

$$
R_{i n}=\frac{L_{s} g_{m}}{C_{g s}}
$$


$\mathrm{R}_{\text {in }}$ is chosen as 50 ohms.

The value of $L$ is picked and the values of $g_{m}$ and $C_{g s}$ are calculated to give the required $R_{i n}$.

$$
L_{g}=\frac{Q_{L} R_{S}}{w_{o}}-L_{S}
$$

Where $\omega \mathrm{o}=$ centre frequency $=2 \cdot \pi \cdot 2 \cdot 65 \mathrm{E}^{9}=1.665 \mathrm{E}^{10} \mathrm{rad} / \mathrm{sec}$ and so $\mathrm{f}_{\mathrm{T}}$ is given as

$$
C_{g s}=\frac{1}{\omega_{o}^{2}\left(L_{g}+L_{S}\right)}
$$

Therefore $\mathrm{Lg}=11.52 \mathrm{nH}$

\subsection{Calculation of $\mathrm{Cgs}$}

$$
C_{g s}=\frac{1}{\omega^{2}{ }_{o}\left(L_{g}+L_{S}\right)}
$$

Thus $C_{g s}=0.4 \mathrm{pF}$

\subsection{Calculation of Transistor widths}

$$
W=\frac{3 C_{g s}}{2 C_{o x} \cdot L_{\min }}
$$

where $\mathrm{L}_{\min }=0.18$ um and $\mathrm{T}_{\mathrm{ox}}=4 \mathrm{e}-9$, Hence $\mathrm{W}=350 \mu \mathrm{m}$.

\subsection{Calculation of Differential Gain}

$$
\begin{aligned}
& g_{m}=\sqrt{2 K_{p} \frac{w}{L}} I_{d s} \\
& \sqrt{\frac{2.170 \cdot 10^{-6} \cdot 650 \cdot 1 \cdot 1 \cdot 10^{-3}}{.18}}=0.36 \\
& A_{L N A}=\frac{g_{m} \cdot Q_{L O A D}}{C o \cdot 2 \pi \cdot 2 \cdot 65 E 9}
\end{aligned}
$$

So, $\mathrm{A}_{\mathrm{LNA}}=12.56$ and $\mathrm{A}_{\mathrm{LNA}}(\mathrm{dB})=22.72 \mathrm{~dB}$. 


\section{Design Summary}

Table 2. Design Summary

\begin{tabular}{lll}
\hline Parameter & Implemented & Unit \\
\hline Lbias & 8.85 & $\mathrm{nH}$ \\
\hline $\mathrm{Lg}$ & 11.5 & $\mathrm{nH}$ \\
\hline $\mathrm{W}(\mathrm{M} 1, \mathrm{M} 2, \mathrm{M} 3$ \& M4) & 350 & $\mu \mathrm{m}$ \\
& & \\
\hline Ls & 5 & $\mathrm{nH}$ \\
\hline
\end{tabular}

After calculations for various parameters, the component values were taken and simulations were carried. Few of the results were not satisfactory, hence near suitable values were taken and simulation was redone. After repeated simulations those values are listed in table -2 which gives the best result as per the specifications. In few cases the tuning tool available with the software[14], has been tried to get the optimized results. After getting the suitable values of components those values were used and final simulation is done. Hence a small change is observed in the calculated and implemented values. In some cases the optimizer is been applied. At the end satisfactory results were obtained which serves the main purpose of this paper.

\section{Analysis and Simulation Results}

Various analysis' were performed to validate the LNA Design

\section{1. s- Parameter Analysis}

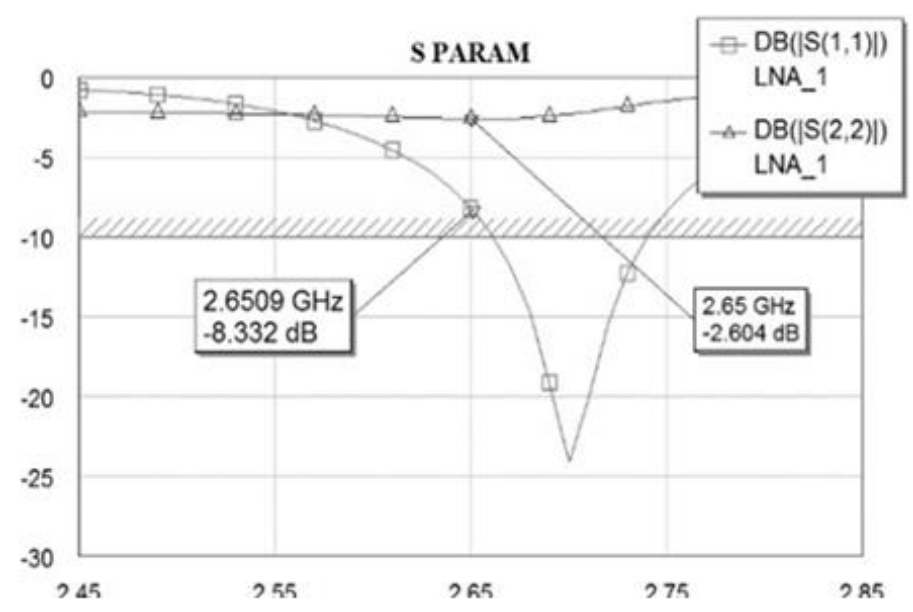

Fig.5. s11 and s22

s- parameters for the design depicts the input and output impedances for the amplifier. The obtained values suites well the design withing the given specifications. The minimum specified values has been shown by the line at the value of $-10 \mathrm{~dB}$. 


\subsection{Gain}

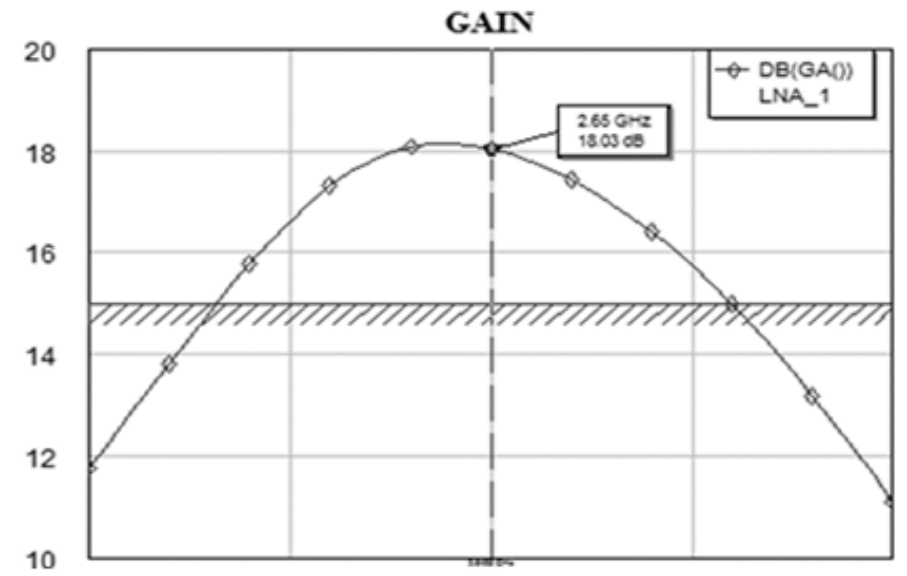

Fig. 6. Frequency Vs Gain

Gain, obtained using the calculated values of parameters was up to the mark, but still there is need of some improvement of design, in terms of improving the overall noise figure of front end, to meet the given specifications.

\subsection{Improved Gain}

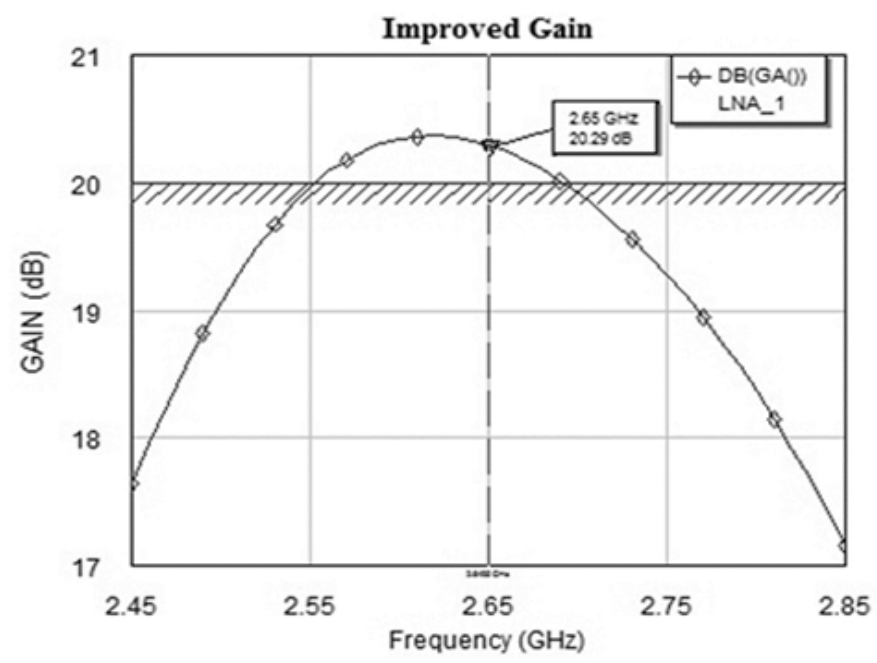

Fig.7. Improved Gain with additional C-S Stage

In order to improve the gain, an additional CS stage [10] [12] have been employed. As a result gain has been improved significantly. The same improvement has been applied in the design to suit the given specifications. 


\subsection{Noise Figure}

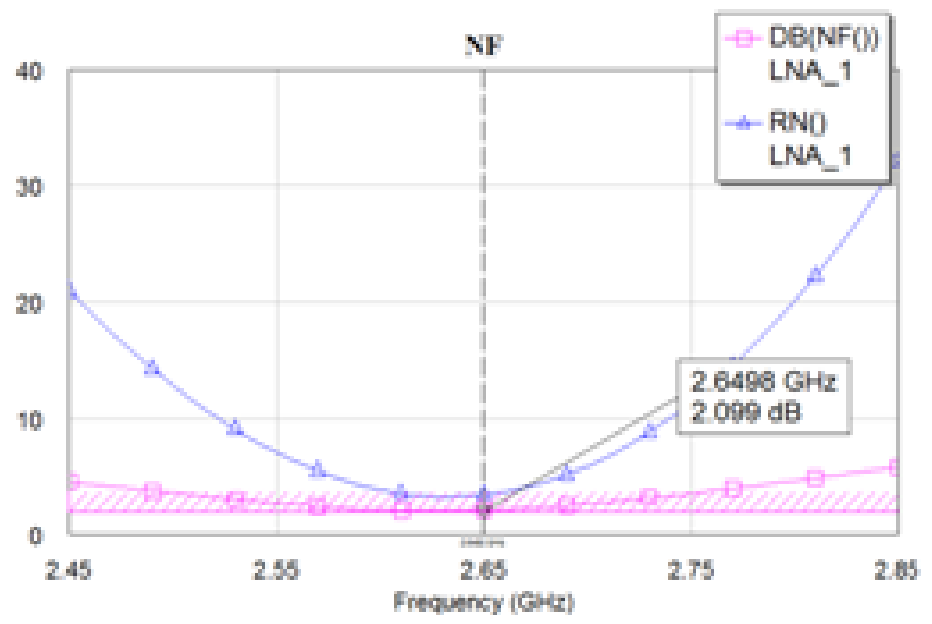

Fig.8. Noise Figure and Noise Resistance(Rn)

As per the Frii's formula, noise figure plays very important role in deciding the overall noise figure of receiver front end. Also LNA should have low noise figure and it should inject less noise to the design.[13]

\subsection{Power Gain}

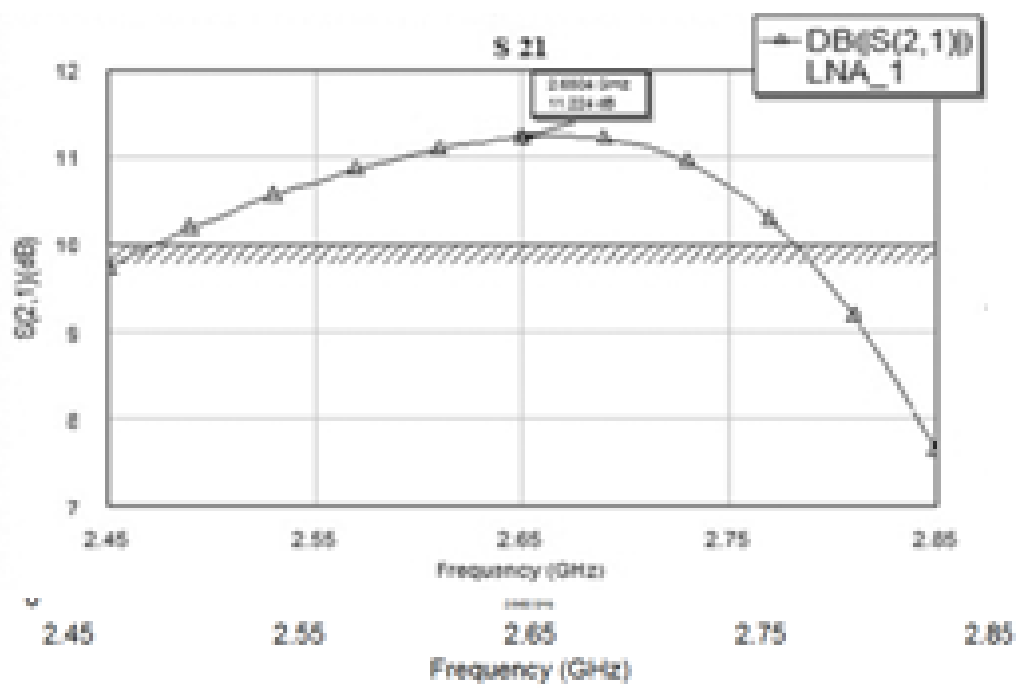

Fig. 9. s-21

Power gain is forward gain $A_{f}$. The value of power gain obtained is well above the specifications. 
5.6. Bias Inductance Vs. Gain

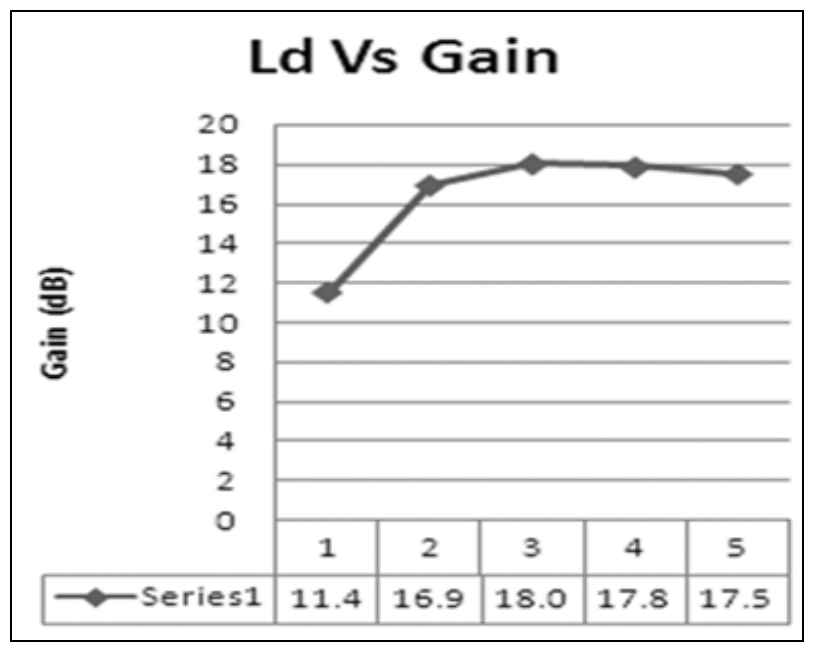

Fig.10. Variation of Gain with Load Inductance(Ld)

Dependence of gain with $L_{d}$ i.e. load inductance is shown. From the results it can be seen that gain of LNA largely changes with the change in load inductance.

\subsection{Lg Vs. Noise Figure}

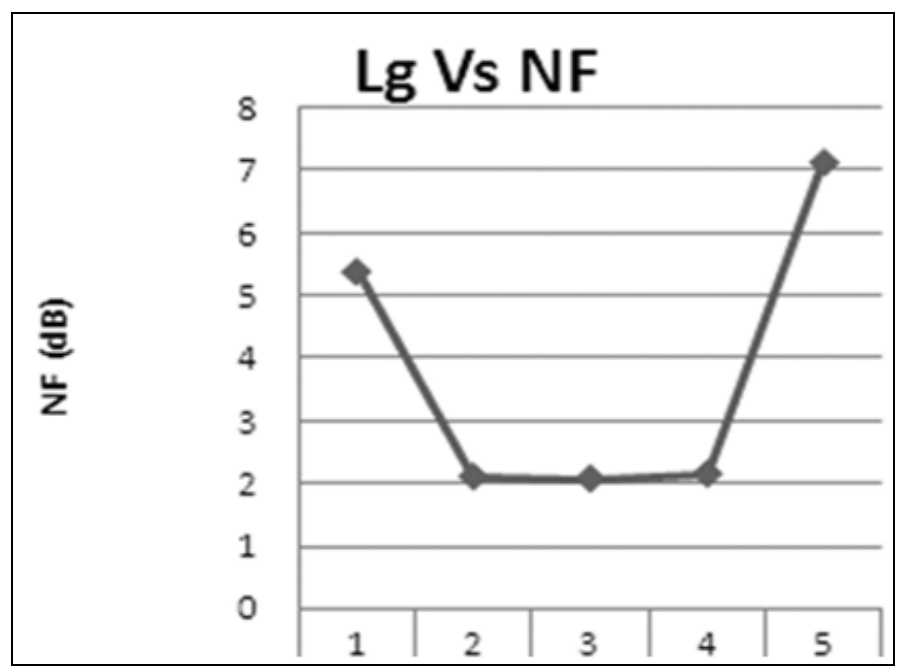

Fig.11. Variation of Noise Figure with Lg

It can be seen that noise figure depends on gate inductance as can be seen. With a small change in Lg noise figure can be changed. Also, gate inductance, in combination with Ls plays very important role in deciding the overall noise figure. 


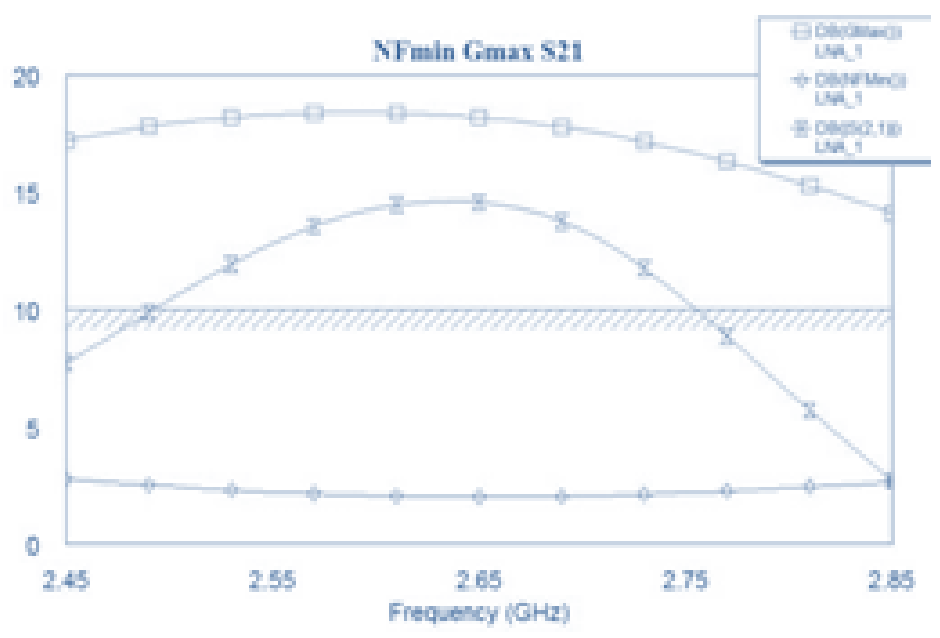

Fig.12: Trade off for NFmin,Gmax and s21

Figure 12 shows the trade off between minimum noise figure, maximum stable gain [9] and power gain of amplifier. Looking at the results it can be seen that variation in power gain and noise figure is linear with respect to frequency, which is well suited for the design.

\section{Summary of Results}

Table 3. Result Summary

\begin{tabular}{llll} 
Parameter & Specifications & This Work & Unit \\
\hline Voltage Gain & $>20$ & 20.29 & $\mathrm{~dB}$ \\
\hline Noise Figure & $<3.5$ & 2.066 & $\mathrm{~dB}$ \\
\hline Power gain & $>10$ & 11.24 & $\mathrm{~dB}$ \\
\hline Load Capacitance & 0.4 & 0.4 & $\mathrm{pF}$ \\
\hline
\end{tabular}

Table 3 shows the result summary of the design. Load capacitance in combination with load inductance gives the value of gain, which meets the specifications. Noise figure is well within the range so that overall receiver front end noise is reduced.

\section{Conclusions}

Simulation for circuit is done using AWR's Microwave Office Tool and different plots are obtained for each of the analysis. Most of the specifications are met in terms of Gain and Noise Figure, which are the main focus of this paper. 


\section{References}

[1] Bosco Leung, "VLSI for Wireless Communication “, Pearson Education Pvt. Ltd, Singapore 2003

[2] J P Silver "MOS Differential LNA Design ", RFIC Journal.

[3] Thomas H. Lee "The Design of CMOS Radio-Frequency Integrated Circuits" 2004.

[4] B.Razavi "RF Microelectronics" Upper Saddle, NJ.

[5] A.Dao "Integrated LNA and Mixer Basics", Application Note 884, National Semiconductors, 2004.

[6] Lab Assignments, Linköping University, Sweden.

[7] Srikanth Arekapudi "Analysis and Design of CMOS Wide-Band Low Noise Amplifiers", Stanford University, August 2004.

[8] Jan H. Mikkelsen, "Front-End Architectures for CMOS Radio Receiver".

[9] Motorola Inc. “Advance Information - Low Power DC-1.8 GHz LNA, Mixer and VCO”, Rev 1, 1998.

[10] Armando Ayala Pabon, Elkim Roa, Wilhelmus Van Noije "An RF CMOS LNA and Mixer merged design strategy", Design and Research Group on IC, Industrial University of Santander.

[11] Xuezhen Wang \& Robert Weber "Design of a CMOS Low Noise Amplifier (LNA) at $5.8 \mathrm{GHz}$ and Its Sensitivity Analysis", Iowa State University, 2004.

[12] Wan -Rone Liou, Mei-Ling Yeh, Chum-An Tsai, Shun-Hsyung Chang "Dedign and Implementation of a low-voltage 2.4 GHz CMOS RF Receiveer Front- End For Wireless Communication", Journal of Marine Science and Technology, Vol. 13, No. 3,pp. 170-175, 2005.

[13] M Sumathi, s Malarvizhi "Design Analysis and Comparitive Study of RF Receiver Front -Ends in 0.18um CMOS”, The IUP Journal of Electrical nad Electronics Engineering, Vol. V, No. 1, 2012.

[14] Applied Wave Research Inc., VSS 2006 Application Notes, 2006.

[15] Clay Couey “2.4 GHz LNA Project "MMIC Design.

\section{Authors Profile}

R.K.Lamba received his B.E. (Electronics) from Nagpur University, Nagpur and M.Tech. (VLSI Design) from Nirma University, Ahmedabad. His area of interest includes RF CMOS Design, Electromagnetics and Analog Design $\mathrm{He}$ is currently the research student at Kadi Sarva Vishwavidyalaya, Gandhinagar.

Dr.C.H. Vithalani received his B.E. (EC) and M.E.(Electronics Engineering) from DDIT Nadiad and Ph.D. from Gujarat University. He is Assoc. Prof. and Head, E\&C Department, Government Engineering College, Rajkot. He worked with Hindustan Conductors Ltd. and Dept. of Telecommunications. He received Research Fellowship from Education Department and Working as a TEQIPCoordinator at GEC Rajkot and as Zonal officer of ACPC (Admission Committee) in west zone. He is Guiding $8 \mathrm{PhD}$ Students at KSV University and Gujarat Technological University

How to cite this paper: R.K.Lamba, C.H.Vithalani,"RF CMOS Low Noise Amplifier Design-A Case Study", IJWMT, vol.4, no.5, pp.14-24, 2014.DOI: 10.5815/ijwmt.2014.05.02 